\title{
Application of Fuzzy Set Theory in the Distribution of Information Flows of a Network Information System
}

\author{
Alexander V. Rubtsov \\ rubtsov.lpi@mail.ru \\ Department of Basic Disciplines \\ Lesosibirsk Pedagogical Institute - branch of Siberian Federal University, \\ Lesosibirsk, Russia
}

Violetta V. Levshina

levshina.rssust@rambler.ru

Department of Quality Management, Standardization, Documentation Management Reshetnev Siberian State University of Science and Technology, Krasnoyarsk, Russia

Svetlana V. Mamaeva

mamaeva.lpi@rambler.ru

Department of Basic Disciplines

Lesosibirsk Pedagogical Institute - branch of Siberian Federal University, Lesosibirsk, Russia

Ludmila N. Khramova

khramova.lpi@rambler.ru

Department of Higher Mathematics, Computer Science and Natural Science

Lesosibirsk Pedagogical Institute - branch of Siberian Federal University, Lesosibirsk, Russia

Igor V. Khramov

khramov.lpi@rambler.ru

Department of Basic Disciplines

Lesosibirsk Pedagogical Institute - branch of Siberian Federal University, Lesosibirsk, Russia

\begin{abstract}
This study's subject represents the processes of functioning of information flows in a network information system through the use of fuzzy sets. It considers the possibilities of fuzzy sets in the information flows distribution problems of the Network Information System by the development of mathematical tools. In the theoretical part of the study, the main issue was the description of the fuzzy approach. In the empirical part of the study, the main concern was finding out the reasons for the fuzzy approach's advantages. The main results of the study: proof of the identity between the functioning of the analytical data; held the solution of the optimization problem based on the formulated analytical models; comparison of the usage of fuzzy logic with a different statistical method; demonstrated the advantage of fuzzy
\end{abstract}


approach in modeling processes network information system. The results can be applied in the field of information systems and technologies.

Keywords: information systems, network information systems, information flows, fuzzy sets, mathematical modeling

\section{Introduction}

Any organization is characterized by a constant change process, explained by the creation and application of new methods in all areas of human activity. The rise of pervasive information and communication technologies seen in cyber-physical systems, the internet of things, and blockchain services has been accompanied by a tremendous growth in the size and complexity of systems [1]. At present, most various organizations' work is mainly associated with the use of information systems. The organization's success depends on the functioning of the latter and on the degree of correspondence between the problems they solve.

The primary function of the information network system is to provide integrated information space. Therefore, the primary vector of development in this area is studying the mechanisms of distribution and competition of information. The story of the information space largely depends on a quantitative change in the amount of data and increasing the complexity of the processes included in it [2].

By information network system (INS) is usually understood as a complex hardware and software structure that includes ergatic, technical, or hardware elements and is included directly in a computer system or computing machine for data transmitting, storing, and processing [3]. The INS has many advantages from modularity, reduced complexity, and memory requirements [4].

Considering modern sources of INS modeling, we can say that the most widely used technology for providing a set of computing resources or their logical combination is implementing user operations on a PC. Currently, researchers have identified many systems that consist in the ability of a particular type of signs to be replaced by characters concerning another class, the capabilities of which are focused on identifying many parameters and not just solving the problem of analyzing the configuration of the studied system [5].

The set of methods and techniques for organizing information processes in the INS is the content of forming an information resource. The INS's structural composition and its direct impact on creating and maintaining an integrated information space indicate an incredibly important component that provides all the fundamental processes of a network information system - the information flow.

The information flow is a tool of the logistics system. A few measures of information flow have been proposed previously in the literature, and actions of directed causal influence are currently being used as a heuristic proxy for information flow. However, there is no rigorous treatment of the problem with formal definitions and clearly stated assumptions. The process of defining information flow is often conflated with the issue of estimating it [6]. A modern way of solving the above problem is to make it language-based. That is: to enrich a programming language with features that specify or control the flow of data so that 
the programs we write are correct [7]. The determining area of importance of information flows mainly to the management domain. It is impossible to build an effective management system without a well-ordered flow of information through its transmission channels. Information flows are the basis of the information system of any organization. Information flows of a course represent the functional and structural organization of the studied object in terms of decision-making mechanisms within the system.

Since any organization's information system refers to the totality of subjects and means of transmitting various types of data, the INS, given the vector of technological exchange of information, is one of the main elements of the structure of the information system. In the INS process, the main difficulties arise when the need for analyzing and monitoring changes in information flows increases. Information resources are dynamic by nature. Changes in external conditions that border the INS's functioning and the untimely receipt of the information lead to several negative consequences. In particular, this is a significant problem for production facilities, which use network systems as a basis for their computer-aided data management. "In those cases, when the requirements for the process of functioning of information flows inside the INS are forced to change constantly, the efficiency of the INS rapidly decreases" [1]. This idea explains the relevance of the search for solutions for a more effective solution to the distribution of information flows in the INS.

The object of this article is the functioning of the information network system.

The study's subject is the presentation of the processes of functioning of information flows in the INS through the use of fuzzy sets.

This article's purpose is to consider the possibilities of fuzzy sets in information flows distribution problems of the INS by the development of mathematical tools.

\section{Literature Review}

Researchers study the distribution of information flows from different points of view. Thus, Gromov considers the process of distribution of information flows in a network information system [4]. Minchenko highlights the streaming networks' performance parameter [8]. Many authors also consider the principle of building structures for the distribution of information flows; in particular, Osin highlights methods for modeling network information systems and the usage of fully polynomial approximation schemes for solving multi-product flow problems [5]. Other authors present a presheaf theoretic approach to the construction of fuzzy sets, which builds on Barr's description of fuzzy sets as sheaves of monomorphisms on a locale [9] and propose definitions of limits of sequences of fuzzy sets, and limits and derivatives of fuzzy set-valued mappings [10]. Jezewski, Czabanski, and Leski defined the real possibilities of describing fuzzy sets and operations on them [11]. Also, other researchers, such as Andrew J. Blumberg and Michael Lesnick when establishing homotopy-theoretic foundations for topological data analysis (TDA), introduced and study homotopy interleavings between filtered topological spaces using fuzzy sets' method as a basic [3]. 
Previously, the distribution of INS information flows using fuzzy set methods was not considered in detail. Moreover, the usage of fuzzy logic is currently limited to modeling linguistic variables and is not widely used in the field of information systems [12]. However, the distribution of INS information flows refers to optimization tasks in conditions of uncertainty.

When characterizing the work of INS, most researchers also highlight an element of uncertainty, which appears due to the following reasons:

- the impossibility of accurate measurements of information flow parameters;

- the use of values close to average in the qualitative and quantitative measurement of information flows;

- a significant difference in the values of the transmitted flows from the average of their values;

- a constant change in the bandwidth capacities of the elements of the INS structure caused by the influence of external factors.

The above-listed explains that the INS parameters cannot be determined under predetermination conditions since the requirements imposed by the user on the transmission of information flows are initially unknown [11]. Moreover, these factors determine the specifics of modeling the distribution of information flows in the INS. Thus, the researchers found that "the use of graph theory in conjunction with the theory of fuzzy sets" seems to be the most accurate way to build analytical models of INS functioning that can reflect the non-determinism of ongoing processes [11].

Information properties of a large set of distributed objects differ from the model object's properties, both quantitatively and qualitatively [13]. Considering the functioning of information flows in the INS, the information resource itself acquires many properties, which should be reflected in the mathematical model of processes. The main difficulty in distributing information flows is the existing feature of the basic model for the analysis of functioning information flows, which includes two different levels: low and high, which means, respectively, open and classified information. The information should not be allowed to flow from high to low variables. Also, before direct modeling of INS information flows, it is necessary to monitor procedures with high information complexity. So, it becomes possible to identify the following problems:

- The procedure has more than one function.

- The procedure is a stress point in the system (with information traffic).

- The procedure has excessive functional complexity.

High informational complexity implies possible advanced testing or redesign procedures. These parameters must be taken into account when modeling, which complicates the use of traditional logic.

In terms of INS, it is worth noting that "one of INS's main properties is their topological dimension Dc" [13]. Many of INS properties depend on the dimension measurement Dc, which is expressed in a standard form as a functional relation of the type "network quality = $\mathrm{f}(\mathrm{Dc})$ ". When considering "the functioning of information flows in the INS in a topological dimension, it becomes possible to 
quantitatively express the properties of the network and find the general information laws of the movement of data flows" [13].

In addition to considering information flows in the dimension determined by the most important invariant of the INS topological space (coverage), there are entirely polynomial approximation schemes used as a group of effective methods computing schemes. A completely polynomial approximation scheme is considered an algorithm for solving combinatorial problems. The option differs from the optimal one by no more than an individual tolerance, at which the level of accuracy acts as a new parameter [5].

Linear programming methods, albeit with significant labor costs, can solve the problems of distributing information flows in the INS. However, in the practical application of such a methodology, it becomes impossible to consider many parameters, which, in turn, leads to enormous miscalculations. Thus, the importance of using methods that could be characterized by the ability to find close to optimal values of variables in a given accuracy is growing.

Human thinking is often associated with fuzzy information originating from initially inaccurate concepts formulated by a human being. Moreover, diversity measures can be computed over different types of data and in a multitude of contexts. Access to data contracts or representations of different real phenomena has allowed for an explosive extension of quantitative studies' reach in many disciplines [14]. The abilities of traditional computational methods developed based on the classical theory of sets, and two-valued logic cannot cope with unreliable and incomplete information. They cannot give expert conclusions on these parameters. In particular, this is a problem in the mathematical modeling of decision-making processes based on various data types.

Today, the managers of production facilities, where the functioning of the INS is the main technical parameter, are in search of flexible IT solutions for managing information circulating within the facility. Thus, today it is customary to use additional modules of already established planning systems. However, in the absence of such an opportunity, computer programs for quick calculations are taken as the basis [10]. Nevertheless, greater interest in the top management of facilities is observed towards the use of fuzzy logic methods in distributing information flows in the INS of a facility since it is impossible to obtain accurate measurements of the values of information flows. The importance of the volumes of transmitted the information flows obtained in this way instantly differs significantly from the average cost.

The advantage of using fuzzy logic methods is justified by interpreting the concept of "truth." So, by "truth," it is customary to understand any linguistic variable that takes the meaning of phrases from natural or artificial languages [10]. So, when creating a mathematical model of any process, it becomes possible to consider more accurate indicators, which is especially important when solving problems with information flows. The accuracy then determines compliance with all the main parameters and principles of the functioning and distribution of information flows. 


\section{Research Methods}

We specified the linguistic variable $(x, T, U, G, M)$. Where $x$ is the name of the variable; $T$ is a term set, each element of which (term) represents as a fuzzy set on a universal stage $U ; G$ - syntax rules, often in the form of grammar, generating the name of the terms; $M$ - semantic rules defining membership functions of fuzzy terms generated by the syntactic rules $G$ " [10]. Using a linguistic variable in modeling makes it easier to implement the decision-making process.

Unlike classical sets, fuzzy sets do not have a clear boundary. The transition from a state of membership to a group to going beyond its borders is characterized by membership functions that allow using fuzzy logic methods to observe flexibility in modeling the linguistic expressions used. Moreover, fuzziness arises not because of the set's constituent element's randomness but because of the uncertain and inaccurate nature of abstract thoughts and concepts [15].

The fuzzy approach does not intend to replace the most straightforward analysis in search of reasonable accuracy; on the contrary, it facilitates the problem of formulating inaccurate restrictions [16]. This fact is why as a result of the integration of approaches from the point of view of set theory and approaches from the point of view of classical programming, it becomes possible to create new methods in managing information flows.

The forms of fuzzy description of information "at input" in management and decision-making problems may vary. It indicates significant differences in the mathematical formulations of the fuzzy mathematical programming problems.

Before moving on to fuzzy modeling of the distribution of information flows in the INS, it is necessary to explain the main concepts and operations inherent in problems solved within fuzzy sets. It is customary to denote any arbitrary set X. A fuzzy set is A defined by using the form's membership function as follows: $\mathrm{X} \rightarrow$ $[0,1] \mu \mathrm{A}$. The value $\mu \mathrm{A}(\mathrm{x})$ is a number whose value satisfies the interval $[0,1]$.

The equality $\mu A(x)=1$ indicates the exact membership of $x$ to the set $A$; the equality $(x)=0 \mu A$ shows the exact non-membership of $\mathrm{x}$ to A. For a standard set, the membership function takes the form as follows:

$$
\mu_{Y}=\left\{\begin{array}{l}
0, x \notin Y \\
1, x \in Y
\end{array}\right.
$$

Provided that the fuzzy set $A$ is normalized, i.e., there exists an element x such that $\mu A(x)=1$ [17].

Let's take two arbitrary fuzzy sets for $A$ and $B$. Membership functions, by definition, will give the result of the operations of combining, intersecting, and complement $A$ on fuzzy sets, which we can express as follows:

$$
\begin{gathered}
\mu_{A \cup B(x)}=\max \left(\mu_{A}(x), \mu_{B}(x)\right), \\
\mu_{A \cap B(x)}=\min \left(\mu_{A}(x), \mu_{B}(x)\right), \\
\mu_{\bar{A}}(x)=1-\mu_{A}(x)
\end{gathered}
$$

Before implementing fuzzy information about the ratio of the limiting number (bandwidth capacity) of structural elements, the volume of transmitted information flows. Several requirements for them in the INS, according to the theory of fuzzy 
sets, fuzzy $L R[11]$ numbers of the form $A L R=(A M, A L, A R)$ are used, where $A M-$ mode and $A L, A R$ are the fuzziness coefficients of the $L R$-number $A L R$.

When performing operations on fuzzy numbers of the form $L R$, it is necessary to note the identity between the functioning analytic representations of the fuzzy number forms $L(y)$ and $R(y)$. Thanks to them, it becomes possible to bring the membership functions (5) as close as possible to the observed values of the INS parameters [18], where $L(y)$ and $R(y)$ are analytical models for representing the shape of a fuzzy $L R$ number:

$$
\mu\left(x, A_{L R}\right)=\left\{\begin{array}{l}
L\left(\frac{A_{M-x}}{A_{L}}\right) \text { at } x \leq A_{M} \\
R\left(\frac{x-A_{M}}{A_{R}}\right) \text { at } x \geq A_{M}
\end{array}\right.
$$

To find as close as possible to the optimal values of the variables in a given accuracy as functions $L(y)$ and $R(y)$ Osin proposed an analytical model for representing the form of a fuzzy $L R$-number of the type indicated later in this study as - (6), which allows one to build numbers that have many ways of membership functions; besides, the model proposed by Osin can replace other used functions [5]:

$$
F(x, p 1, p 2)=\left(\max \left(0,1-|x|^{p 1}\right)\right)^{p 2}
$$

where $\quad p 1, p 2-$ function parameters, at $p 1>0, p 2>0$.

When distributing information flows in the INS, it is necessary to consider that each output and input pair has many methods that can identify the maximum transmission efficiency [11]. The INS structure, in this case, will become supreme. Since it is precisely on this parameter that the dependence of the methods for fulfilling each pair's requirements at different levels will be determined, thereby ensuring conflict-free transmission when an increase in one pair's transmitted information flow does not reduce that for the other pair. To determine these levels, Osin proposed to "consistently solve the optimization problem" [5], the author's analytical model of which has the following form $F(\tilde{f}) \rightarrow \max$ :

$$
F(\tilde{f})=k_{1} F 1(\tilde{f})+k_{2} F 2(\tilde{f})+k_{3} F 3(\tilde{f})+k_{4} \theta(7)
$$

in accordance with the restrictions:

$$
\begin{gathered}
F 1(\tilde{f})=\frac{\sum_{i=\overline{1, p}}\left(N \max _{i}-\left|\widetilde{f_{l}}\right|\right)}{\sum_{i=\overline{1, p}}\left(N_{\max _{i}}-1\right)}, \\
F 2(\tilde{f})=\sum_{i=\overline{1, p}}\left(\operatorname{Lmax}_{i}-\frac{\sum_{j=1,\left|f_{i}\right|} L\left(\widetilde{f_{l, j}}\right)}{\left|\widetilde{f_{l}}\right|}\right), \\
F 3(\tilde{f})=\frac{\sum_{i=\overline{1, p}} \sum_{j=1, \mid \widetilde{f_{l}}} \widetilde{f_{l, j}}}{\sum_{i=\overline{1, p}} \widetilde{d_{l}}}, \forall \mathrm{e}: \sum_{P: e \in P} \widetilde{f}(P) \widetilde{c}(e), \\
0 \leq F 1(\tilde{f}) \leq 1,0 \leq F 2(\tilde{f}) \leq 1,0 \leq F 3(\tilde{f}) \leq 1
\end{gathered}
$$

where $\quad k_{1}-k_{4}-$ weighting factors set by the decision maker;

$k_{1}+k_{2}+k_{3}+k_{4}=1$;

$F 1(\tilde{f}), F 2(\tilde{f})$ are estimated $\tilde{f}$ by the number of information flows and nodes in them;

$\operatorname{Nmax}_{i}$ and $\operatorname{Lmax}_{i}$ - the maximum possible number of components and the path length for the information flow $i$;

$\left|\widetilde{f}_{l}\right|$ - a number of components for $i$ flow in distribution $\tilde{f}$; 
$L\left(\tilde{f}_{i, j}\right)$ - a path length of $j$ component;

$F 3(\tilde{f})$ - the total fulfilment of the requirements of gravitating pairs;

$\theta$ assesses the lower limit of security requirements;

$\tilde{c}(e)$ - the capacity of arc $e$;

$P$ - the information flow transmission path;

$\tilde{f}(P) \sim-$ a volume of information flow transmitted along the path $P[18]$.

The analytical models for representing the type of a fuzzy LR number shown in formulas (6) and (7) of this article can formalize non-deterministic data on the parameters of information flows in the INS in the form of LR numbers and then determine the degree of efficiency of their distribution. It considers all the parameters formalized by the model based on fuzzy logic's concepts and properties [19]. Namely, the structure of information flows, which means managing complex problems, such as load balancing, processor synchronization, and access to information, are taken into account.

Since fuzzy sets are a generalization of the classical ones known to us, the operations should be reduced to the ones we are familiar with in particular cases. Functions must be defined outside of the theory of fuzzy mathematics itself and, therefore, its adequacy cannot be verified by means of the approach itself. Based on the essence of subjective evaluations, the function can only be constructed based on expert assessment. In our task evaluating the distribution's effectiveness as a fuzzy composite option is based on the work by Geske [20]. The transition to formulas suitable for fuzzy sets is made using the expansion principle. Applying the extension principle to arithmetic operations and trapezoidal fuzzy numbers, we get the following rules for addition and subtraction (Figure 1).

\section{a triangular number}

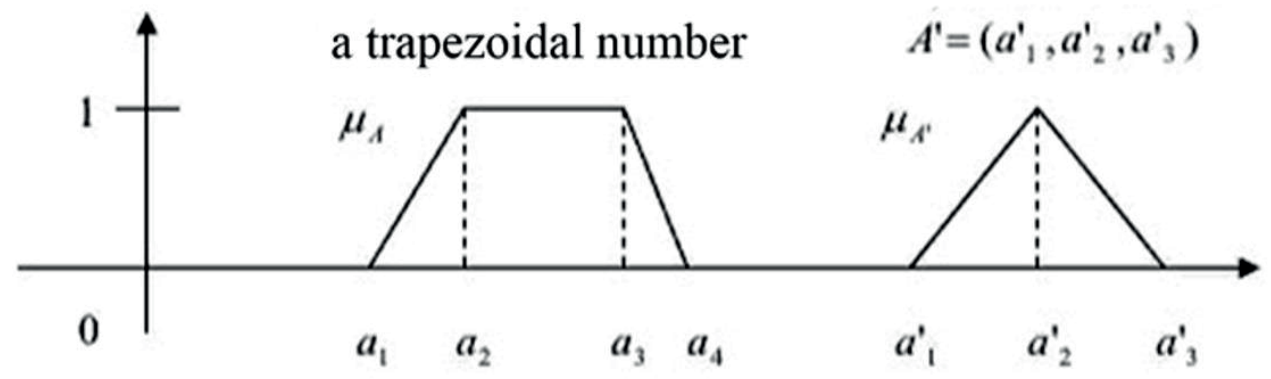

Figure 1. Trapezoidal and triangular numbers.

$$
\begin{aligned}
& \left(a_{1}, a_{2}, a_{3}, a_{4}\right)+\left(b_{1}, b_{2}, b_{3}, b_{4}\right)=\left(a_{1}+b_{1}, a_{2}+b_{2}, a_{3}+b_{3}, a_{4}+b_{4}\right) \\
& \left(a_{1}, a_{2}, a_{3}, a_{4}\right)-\left(b_{1}, b_{2}, b_{3}, b_{4}\right)=\left(a_{1}-b_{4}, a_{2}-b_{3}, a_{3}-b_{2}, a_{4}-b_{1}\right)
\end{aligned}
$$

The use of fuzzy logic elements in calculus tools is not limited to the values "true" and "false." Here we see the main advantage of fuzzy logic in creating an empirical knowledge model that determines which control actions should be performed for a given input data set. 
To determine the effectiveness of using fuzzy sets, consider distributing the information flow of a network information system in three stages. In this problem, unlike the previous calculations, the fuzzy set method will be used as an auxiliary method since this type of situation is optional (Figure 2).

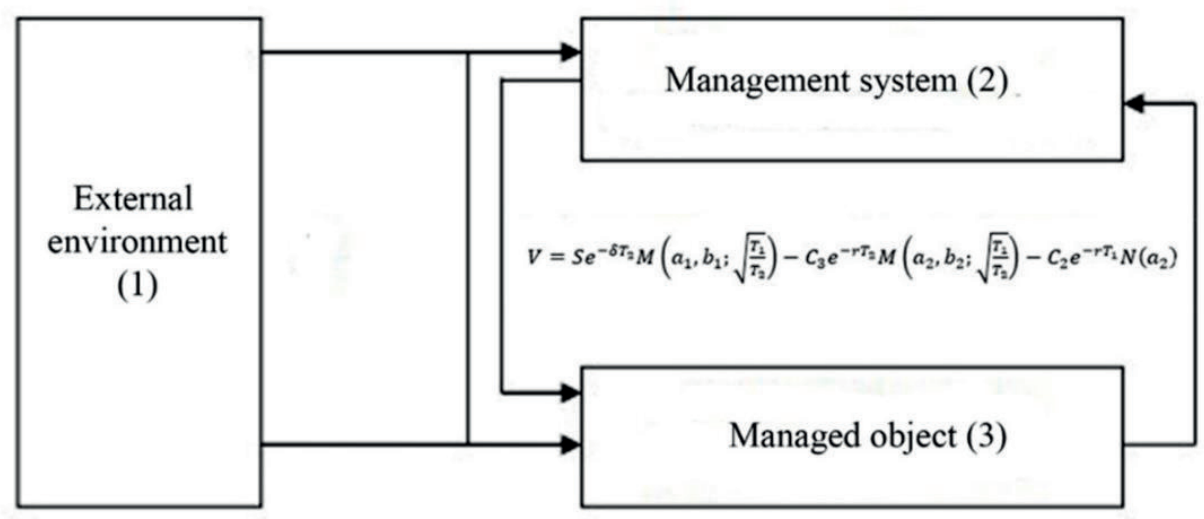

Figure 2. The illustration of the problem.

It is assumed that at the beginning of each stage, an absolute return of information is made (costs); we denote the value of the return of information at each stage $C 1, C 2, C 3$, respectively. In addition to distributing existing data, the information flow receives an additional data set during each stage. This condition is vital when moving to each next step. Let $S$ denote the receipt of information leading to the beginning of the third stage of distribution. It is assumed that $C 1, C 2, C 3, S$ are fuzzy numbers obtained by expert analysis. The fuzzy variables must be centered to perform calculations. It makes it possible to reduce operations to addition, subtraction, and multiplication. When switching to operations for fuzzy sets, this property is of great importance for further visualization of the obtained data under homotopy topology conditions [17]. Let $T 1, T 2$ be the time for passing the first and second stages, and $r$ be the parameter that determines the information flow's efficiency.

Now we should move on to make formulas for solving the problem. Based on the above analysis of the issues, it is necessary to distinguish the following properties: the identity between functioning analytical representations (5); the INS structure parameter determines how to fulfill the flow requirements at different levels, thus ensuring conflict-free transmission (7). We consider the following task as the task of effective distribution the information flow of a network information system. Thus, we calculate the INS information flow distribution efficiency using our formula based on the real option's method. Using the real options method, it becomes possible to consider improving specific parameters at the stage of evaluating the effectiveness of a particular technology. Thus, we calculate the efficiency of the INS information flow distribution using our formula, which is based on the real option's method, where we are going to estimate an evaluating the current distribution of information flow: 


$$
V=S e^{-\delta T_{2}} M\left(a_{1}, b_{1} ; \sqrt{\frac{T_{1}}{T_{2}}}\right)-C_{3} e^{-r T_{2}} M\left(a_{2}, b_{2} ; \sqrt{\frac{T_{1}}{T_{2}}}\right)-C_{2} e^{-r T_{1}} N\left(a_{2}\right)
$$

where:

$$
\begin{gathered}
a_{1}=\frac{\ln \left[E(S) / S^{c}\right]+\left(r-\delta+\frac{\sigma^{2}}{2}\right) T_{1}}{\sigma \sqrt{T_{1}}}, a_{2}=a_{1}-\sigma \sqrt{T_{1}},(11) \\
b_{1}=\frac{\ln \left[E(S) / E\left(C_{3}\right)\right]+\left(r-\delta+\frac{\sigma^{2}}{2}\right) T_{2}}{\sigma \sqrt{T_{2}}}, b_{2}=b_{1}-\sigma \sqrt{T_{2}},
\end{gathered}
$$

$$
\sigma=\frac{\sqrt{\operatorname{Var}(S)}}{E(S)}-\text { a statistical indicator that characterizes variability, }
$$

$\delta=\frac{E\left(C_{1}\right)}{E(S)}-$ the amount of information received at each of the three stages,

$N(a)$ - the standard normal distribution function,

$E(S)$ - the expected value of the distribution efficiency of information flow,

$M(a, b ; \rho)$ - is the cumulative function of the double normal distribution with the correlation coefficient $\rho$ (that is, the cumulative function of a pair of standard normally distributed random variables, the correlation between which is equal to $\rho$ ), and the critical value of the information flow distribution INS $S^{c}$ is the root of the following equation:

where:

$$
S^{c} e^{-\delta\left(T_{2}-T_{1}\right)} N\left(c_{1}\right)-E\left(C_{3}\right) e^{-r\left(T_{2}-T_{1}\right)} N\left(c_{2}\right)-E\left(C_{2}\right)=0,(13)
$$

$$
\begin{gathered}
c_{1}=\frac{\ln \left[S^{c} / E\left(C_{3}\right)\right]+\left(r-\delta+\frac{\sigma^{2}}{2}\right)\left(T_{2}-T_{1}\right)}{\sigma \sqrt{T_{2}-T_{1}}},(14) \\
c_{2}=c_{1}-\sigma \sqrt{T_{2}-T_{1}},(15)
\end{gathered}
$$

Thus, it becomes possible to calculate the efficiency of the INS information flow distribution that passes through the three stages of the problem condition. Let $C_{1}=$ $(40,50,60), C_{2}=(280,300,320), C_{3}=(630,700,770), S=(2000,2500,3000)$, these are estimates of the return of information from all three stages as a result of the distribution of the information flow INS by means of fuzzy numbers. Let $T_{1}=3, T_{2}$ $=5, r=5 \%$.

Then

where $\sigma=0.082, \delta=0.02$.

$$
\begin{gathered}
E(S)=2500, \\
\operatorname{Var}(S)=41666.7, \\
E\left(C_{3}\right)=700, \\
\operatorname{Var}\left(C_{3}\right)=816.7, \\
E\left(C_{1}\right)=50 .
\end{gathered}
$$

The critical value is approximately equal to $S^{\mathrm{c}}=971.47$.

Therefore, we have:

$$
\begin{gathered}
a_{1}=7.39, \\
a_{2}=7.25, \\
b_{1}=7.89, \\
b_{2}=7.7 .
\end{gathered}
$$

As a result, we get the following estimate of the information flow distribution INS: 


$$
V=(934.57,1458.72,1982.87)
$$

As a validation of the proposed model, solved using the fuzzy set methodology, it is proposed to use the following scheme (Figure 3).

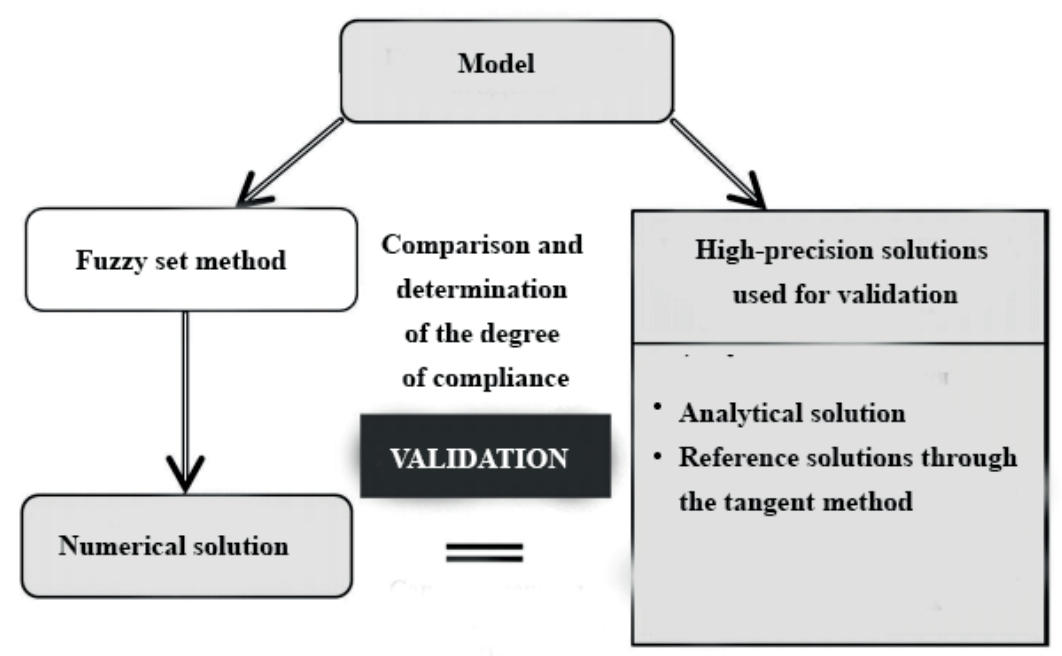

Figure 4. Validation scheme.

Thus, the model's representation and its solution via the fuzzy set method are reflected in the formulas (10-15). It should be noticed that the following process requires analytical analysis when choosing the solution result. We can also note that the solution involves many conditions to be met, which makes it difficult to make changes to the parameters' values, which, due to their fuzziness, can significantly complicate the process of finding solutions.

To compare the results' accuracy, we do the calculation through a numerical solution without introducing fuzzy numbers. Thus, the evaluation of the efficiency of the INS information flow distribution by calculating the efficiency depending on the expected addition of quality data at the end of the third stage is equal to:

$$
V=(850,1450,2050) \text {. }
$$

As for high-precision solutions the tangent method is used (Figure 5). In this method the calculation is performed by using the approximate numbers.

According to Figure 6, we have:

$a-$ the left border of the section

$b-$ the right border of the section

$\varepsilon-$ given accuracy

$i_{\text {max }}$ - a maximum number of iterations

$x$ - the approximate value of the root

$f_{x}-$ the value of the function at $x$

$\delta$ - the achieved accuracy

$i-$ a number of iterations 


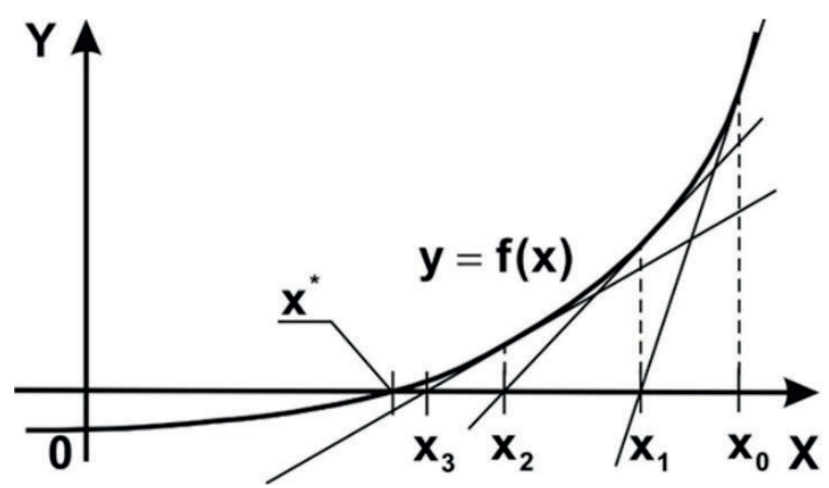

Figure 5. Geometric illustration of the tangent method.

The following algorithm for finding variables is applied to Figure 6.

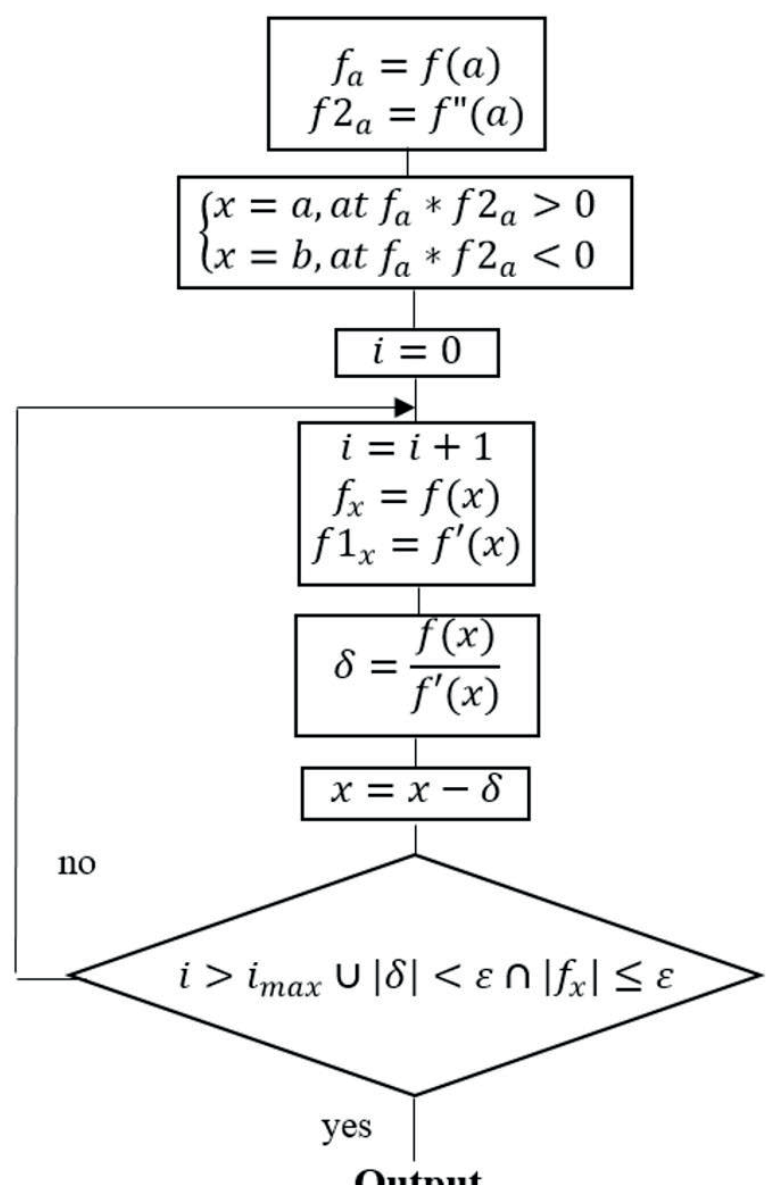

Figure 6. The algorithm of the tangent method. 
According to our problem, we get the following equation:

$$
S \mathrm{c}=V+C_{3} e^{-r T_{2}}+C_{2} e^{-r T_{1}}
$$

It is necessary to present the found parameter $V$ - an indicator of the efficiency of information flow distribution as a function. By entering all the necessary notation, we get the following equation:

$$
f(V)=V+C_{3} e^{-r T_{2}}+C_{2} e^{-r T_{1}}
$$

We use the previous calculations to enter the values of known variables. Using Figure 3, we assume that the separation of the roots is made and there is one root located on the interval $[a, b]$, which must be specified with the error $\varepsilon$.

So, we have $f(a) f(b)<0$.

Define half of the segment $c=1 / 2(a+b)$ and calculate $f(c)$. We check the following conditions:

1. If $|f(c)|<\varepsilon$, then $c$ is the root. Here $\varepsilon$ is the specified accuracy;

2. If $f(c) f(a)<0$, then the root lies in the interval $[a, c]$;

3. If $f(c) f(b)<0$, then the root lies on the segment $[c, b]$.

Continuing the process of half division in the selected subintervals, you can reach an arbitrarily small segment containing the root $\xi$.

Since the interval where the root is located is halved for each iteration, then after $\mathrm{n}$ iterations the interval will be equal to:

$$
b_{n}-a_{n}=1 / 2(b-a) \text {. }
$$

As the root of $\xi$. take $1 / 2\left(a_{n}+b_{n}\right)$. Then the error in determining the root will be equal to $\left(b_{n}-a_{n}\right) / 2$. If the condition is met: $\left(b_{n}-a_{n}\right) / 2<\varepsilon$.

Then the search process ends and $\xi=1 / 2\left(a_{n}+b_{n}\right)$.

The number of steps required to achieve the specified accuracy is determined by the inequality:

$$
h \geq\left(\log _{2} \frac{b-a}{\varepsilon}\right)+1
$$

Calculating the efficiency of information flow distribution, we get the following results (Table 1).

\begin{tabular}{|c|c|c|c|c|c|c|}
\hline No. & $\boldsymbol{c}$ & $\boldsymbol{a}$ & $\boldsymbol{b}$ & $\boldsymbol{f}(\boldsymbol{c})$ & $\boldsymbol{f ( \boldsymbol { V } )}$ & $\boldsymbol{\varepsilon}$ \\
\hline 1 & 0 & 0 & 10 & -4510 & 2500 & 10 \\
\hline 2 & -5 & -5 & 0 & 2500 & -1005 & 5 \\
\hline 3 & -2.5 & -2.5 & 0 & -1005 & 747.5 & 2.5 \\
\hline 4 & -3.75 & -3.75 & -2.5 & 747.5 & -128.75 & 1.25 \\
\hline 5 & -3.125 & -3.125 & -2.5 & -128.75 & 309.375 & 0.625 \\
\hline 6 & -3.4375 & -3.4375 & -3.125 & 309.375 & 90.3125 & 0.3125 \\
\hline 7 & -3.5938 & -3.5938 & -3.4375 & 90.3125 & -19.2188 & 0.1563 \\
\hline
\end{tabular}

Table 1. The iterations of the root.

The estimate calculated without introducing fuzzy numbers was more general: the lower bound of the corresponding fuzzy number differs by $10 \%$, the most 
possible value - by $0.5 \%$, and the upper bound-by $3 \%$. This confirms the idea that using the fuzzy set method in information flow distribution problems allows us to see the most accurate data with an accuracy of 0.01 .

\section{Findings}

Considering the problem of distributing the INS information flows, we highlight the primary definition of the possible efficiency of distribution, implying that specific parameters can change. Using our formula based on calculating cash flows, we were able to get more accurate estimates when using operations with fuzzy sets to obtain numerical values.

The above analysis of the use of fuzzy models in the distribution of information flows in the INS allows us to highlight the following advantages:

- modeling of dynamic problems, the results of various studies, as well as operations on them;

- fuzzy presentation of the formalization of evaluation and comparison criteria;

- the use in the calculation of not only data values, but also their parameters, and the subsequent distribution in the system based on productive models;

- reduction of time and labor costs when modeling complex dynamic systems and comparing them for a given degree of accuracy.

As a result of the research, we have found that fuzzy set methods can form the basis for solving problems of distribution of INS information flows and can be used in conjunction with other methods. In the comparison of traditional technique and fuzzy logic, we have proved the effectiveness of fuzzy methods. Using fuzzy logic and conventional statistical methods in combination, we introduced the variability parameter values and the volume of information. Using centered variables in the solution of the problem to perform further operations inherent in fuzzy sets, we refer to the perspectives of programming these problems in the context of homotopy type theory. Using fuzzy set methods allows getting more accurate results due to the above properties, taking into account all the parameters and restrictions typical for such a phenomenon. Based on international experience, we have determined a way to find the variables that are as close as possible to the optimal values in a given accuracy. Thus, by adding mathematical tools to the study of the distribution of information flows INS, it becomes possible for the expert to see more accurate data on the organization's distribution of information. In the future, these indicators can serve as a basis for improving the organization's information support, thereby ensuring the correct operation of the network. Our method provides a refinement relation and a composition operation that supports both the quality of information and implementation ability. We develop a basic theory for better results. We illustrated the applicability of our idea with an example of information flows' distribution.

Consistent with earlier research, the study supported the hypothesis that using the fuzzy approach is effective. Thus, considering the solution of the problem based on the models proposed by Osin, who pointed attention to the question of matching 
the data under consideration [5], we can prove the identity between the analytical data, as well as the ability to most accurately reflect the parameters in mathematical modeling.

It is also worth mentioning the contributions of other authors, particularly Jezewski, Czabanski, and Leski, who defined the real possibilities of describing fuzzy sets and operations on them [11]. Based on the propositions put forward by these authors, we found that the properties of fuzzy sets can be used as an auxiliary parameter for optional problems. After investigating the issue, we concluded that fuzzy set methods are not widely used to solve the distribution of INS information flows.

Since we were faced with identifying the advantages of using fuzzy set methods in the distribution of information flows of an INS, we first turned to the primary source of theoretical material - the work of Osin [5]. As a result of reviewing the mathematical tools of this methodology, based on the formulas and definitions proposed by Osin [5], we identified the advantages in the conciseness of procedures and opportunities for modeling fuzzy information by description. Further, we assumed that the processing of the essential mathematical tools for solving information flow distribution problems in the INS would not be rational due to subsequent computer modeling problems and presenting more accurate results.

Based on this point of view, we decided to implement the fuzzy set method in conjunction with the main functioning tools. At this stage of the study, we found that using a fuzzy approach is rational when modeling the main variables, which we reflected when solving our problem. The main problem of the distribution of information flows in the INS is the inability to recall many modeling parameters. Our proposed approach, reflected in the problem, removes these limitations and allows us to get more accurate values.

Unlike other researchers, we make it the key to improving the quality of the distribution information. We use the example of our problem to discover that using the fuzzy set method in conjunction with other calculus types helps to get more accurate estimates. Accordingly, we consider this approach to be practical and easy to implement.

In the present study, we confirm the effectiveness of using fuzzy set methods in the distribution of INS information flows by using this method as the main one and as an auxiliary one. Thus, we consider it necessary to improve this field's mathematical tools based on the proposed approach. We suggest integrating already proven methods in conjunction with fuzzy logic methods, which in the future presents the possibility of modeling such problems in modern and accessible programming languages. Thus, in the course of the study, we concluded developing mathematical tools by using fuzzy sets in solving problems of distribution of information flows of INS.

Therefore, we highlight possible prospects for the development and application of the theory of fuzzy sets, which is suitable for different software structures. Summing up the calculations, we can conclude that fuzzy modeling is optimal when the technological processes are of some complexity when analyzing using generally accepted quantitative methods. According to our idea, fuzzy logic provides for the 
availability of mathematical tools to reflect the source information's fuzziness. Also, it allows you to build a model that is as close as possible to reality since, in some cases, the use of fuzzy models makes it possible to formalize the source data with less error.

\section{Conclusion}

Summarizing all the above, the advantage of fuzzy modeling of the processes of distribution of information flows in the INS becomes evident since the determinism requirement present in the INS's functioning is formalized in this particular method. Since, like all electronic streams, electronic information is determined through difference and is also characterized by parameters of variability, interdependence, and time dependence, necessary to organize the distribution of information flows in the INS by taking into account the uncertainty of information (its display in a mathematical model). The importance of observing the INS uncertainty parameter is explained by the possibility of constructing a model that is most closely approximated to reality, which, in turn, is not difficult to perform operations on models. Failure to comply with the uncertainty parameter in the classical theory of probability is a gross omission of any processes' real properties. Many uncertainty factors characterize any of them, particularly the investment project, the research base, the workflow process, and other types of information.

Thus, in this paper, when considering the INS' distribution of information flows, we highlight the following opportunities for applying this technique in management. The results we highlight as the formation of mathematical tools allow us to expand industrial automation capabilities. This idea makes it possible to use existing knowledge to improve technological processes and perform various tasks. For example, for:

- controls (closed or open systems, one or more variables in linear or nonlinear systems);

- configuration parameters of the system in online or offline mode;

- classification and image recognition;

- real-time decision making;

- help operators in making decision or when adjusting the parameters;

- detection and diagnostics of system failures.

A wide range of application options and a natural approach based on the acquired experience make this method the primary mathematical tool that should become the standard for decision-makers.

Due to its relevance, the fuzzy approach provides a broad base for further research of feasible processes without introducing additional software. This idea greatly simplifies the consumption of resources and uses the existing means of information technology as an element of any organization's information system in full. 


\section{References}

[1] E. Bartocci, T. Ferrère, T. A. Henzinger, D. Nickovic, and A. O. da Costa, "Information-flow interfaces," Computer Science, vol. 1, pp. 1-33, 2020. [Online]. Available: http://arxiv.org/abs/2002.06465

[2] C. Shi, and P. S. Yu, Heterogeneous information network analysis and applications. 2017, doi: 10.1007/978-3-319-56212-4.

[3] A. J. Blumberg, and M. Lesnick, "Universality of the homotopy interleaving distance," Cornell University Press, pp. 1-29, 2017. [Online]. Available: http://arxiv.org/abs/1705.01690

[4] Yu. Yu. Gromov, Yu. V. Minin, A. B. Bakhsh, A. V. Klishina, and M. A. Shakhov, "Determination of groups of negative external influences on the network information system that maximize the value of the damage function," Information Security, vol. 2, pp. 269-272, 2014.

[5] V. N. Osin, "Effective distribution of information flows in a network information system based on fuzzy models," Ph.D. dissertation, Tambov, 2014.

[6] P. Venkatesh, S. Dutta, and P. Grover, "Information flow in computational systems," IEEE Transactions on Information Theory, vol. 66, no. 9, pp. 5456 - 5491, 2020, doi: 10.1109/TIT.2020.2987806.

[7] G. A. Kavvos, "Modalities, cohesion, and information flow," Proceedings of the ACM on Programming Languages, vol. 3(POPL), pp. 1-29, 2019, doi: $10.1145 / 3290333$.

[8] L. V. Minchenko, and N. P. Yakovleva, "Application of fuzzy logic in food industry enterprise management," Scientific Journal of ITMO Research Institute. Series 'Economics and Environmental Management, vol. 4, pp. 270-279, 2015.

[9] J. F. Jardine, "Fuzzy sets and presheaves," Compositionality, vol. 1, no. 3, 2019, doi: 10.32408/compositionality-1-3.

[10] M. Kon, and H. Kuwano, "On sequences of fuzzy sets and fuzzy set-valued mappings," Fixed Point Theory and Applications, vol. 2013, no. 1, pp. 327, 2013, doi: 10.1186/1687-1812-2013-327.

[11] R. Czabanski, M. Jezewski, and J. Leski, "Introduction to fuzzy systems," in Theory and applications of ordered fuzzy numbers: A tribute to professor Witold Kosiński, P. Prokopowicz, J. Czerniak, D. Mikołajewski, Ł. Apiecionek, and D. Ślȩzak (Eds.), pp. 23-43, 2017, doi: 10.1007/978-3319-59614-3_2.

[12] C. Shi, Y. Li, J. Zhang, Y. Sun, and P. S. Yu, "A survey of heterogeneous information network analysis," IEEE Transactions on Knowledge and 
Data Engineering, vol. 29, no. 1, pp. 17-37, 2017, doi: 10.1109/TKDE.2016.2598561.

[13] B. Bogaerts, and D. Surinx, J. Van den Bussche, H. Aamer, and E. Ternovska, "Executable first-order queries in the logic of information flows," Cornell University Press, vol. 3, pp. 1-23, 2019.

[14] P. Ramaciotti Morales, R. Lamarche-Perrin, R. Fournier-S'niehotta, R. Poulain, L. Tabourier, and F. Tarissan, "Measuring diversity in heterogeneous information networks," Computer Science, pp. 3-31, 2020.

[15] A. A. Baichenko, "Application of fuzzy logic in the management of food industry enterprises," Scientific Journal of ITMO Research Institute. Series 'Economics and Environmental Management, vol. 3, pp. 35-69, 2014. [Online]. Available:

https://openbooks.itmo.ru/ru/article/10476/primenenie_nechetkoy_logiki_v _upravlenii_predpriyatiem_pischevoy_promyshlennosti.html

[16] A. Yu. Shatalova, and K. A. Lebedev, (2015). "Fuzzy linear programming in the problem of optimal financing of investment projects," Fundamental Research, vol. 9, pp. 35-38, 2015.

[17] G. Allwein, and W. L. Harrison, "Information flow and homotopy theory," Elsevier, vol. 1, pp. 1-38, 2019.

[18] R. E. Kent, "Semantic integration in the information flow framework," Cornell University Press, vol. 1, pp. 1-12, 2018. [Online]. Available: http://arxiv.org/abs/1810.08236

[19] H.-C. Wu, "Duality in fuzzy sets and dual arithmetics of fuzzy sets," Mathematics, vol. 7, no. 1, pp. 11, 2018, doi: 10.3390/math7010011.

[20] R. Geske, "The valuation of compound options," Journal of Financial Economics, vol. 7, no. 1, pp. 63-81, 1979, doi: 10.1016/0304405X(79)90022-9. 